\title{
Scalable Routing in Hybrid Cellular and Ad-Hoc Networks
}

\author{
Ioannis Ioannidis, Bogdan Carbunar \\ Purdue University \\ West Lafayette, IN, 47907 \\ \{ioannis,carbunar\}@cs.purdue.edu
}

\begin{abstract}
-
As wireless ad-hoc networking approaches its maturity, an architecture that can support the massive deployment of such networks has not been established. Hybrid networks are a promising architecture that builds ad hoc, wireless networks around the existing cellular telephony infrastructure. In this paper we present a routing protocol (DST) for hybrid networks that maintains a close to optimal spanning tree of the network with the use of distributed topology trees. DST is fully dynamic and generates only $O(\log n)$ messages per update operation. We demonstrate experimentally that the performance of DST scales excellently with the network size and activity, making it ideal for the metropolitan environment hybrid networks are expected to operate in.
\end{abstract}

\section{INTRODUCTION}

The past decade has witnessed rapid developments in wireless communications, from wireless cellular telephony to ad-hoc networks, wireless LANs and RF networks. Wireless network cards have become affordable and wireless connections are fast enough for users to abandon more traditional networking possibilities, as long as there is a nearby access point. The only factor against an explosion of wireless computer networking is the necessity for an expensive infrastructure that can provide extensive and reliable coverage with sufficient bandwidth.

Currently, the only infrastructure that addresses the above problem is that of cellular telephony. Nevertheless, when a laptop equipped with a wireless network card connects to a base station the same way as cell phones, the bandwidth limitations are severe; the top rate achievable in a cell is $2.4 \mathrm{Mbps}(1 \mathrm{xEV}-\mathrm{DO})$ and the bandwidth drops fast as the device moves away from the base station. Furthermore, the transmission rate can be extremely erratic, making the network unreliable. Upgrading cellular base stations can solve these problems, although it is doubtful that providers will be willing to make such a massive investment.

A solution that grafts ideas from ad hoc networks into cellular technology has started to attract attention. As in ad hoc networks, connections can involve several intermediate relayers. Since wireless LANs offer high throughput (IEEE $802.11 \mathrm{~b}$ offers up to $11 \mathrm{Mbps}$ ), albeit in a limited range, using a web of multihop paths can considerably increase the throughput from the base station to the devices in its cell without requiring modifications in the infrastructure [1].

A simple solution to the problem of multihop path dis- covery in a hybrid network is also described in [1]. An initiator discovers a path to the base station with a breadthfirst search of the network. The disadvantage of flooding the network every time a path is needed is that the traffic generated by the routing protocol can cause severe congestion. Another disadvantage is that when multiple hosts try to find a path to the base station, hosts that have a good downlink rate will be congested as they will be on many paths.

To overcome such difficulties, we propose a routing algorithm that is based on the maintenance of a spanning tree of the network. There are two reasons for using a spanning tree. The first is that, as we show, a maximum spanning tree provides the optimal routing for the next flow from a host to the base station that has to be scheduled. We do not maintain a maximum spanning tree, but our protocol will lazily converge to one. Experiments show that the achieved throughput is consistently over $80 \%$ of the optimal. The second reason for structuring the routing information as a spanning tree is that we can maintain it by generating $\mathrm{O}(\log \mathrm{n})$ traffic for each routing request, where $\mathrm{n}$ is the number of nodes in the network, instead of $\mathrm{O}(\mathrm{n})$, when flooding is used. We achieve this with topology trees [2].

\section{Overview of the Protocol}

\section{A. Spanning Trees}

Consider a hybrid network where no host needs a multihop path to the base station. All links are available to their full capacity and we assume that there is a method to estimate this capacity (for example, hosts periodically ping their neighborhood and measure the response time). If a host A needs the best available path to the base station(BS) and there is an optimal path discovery protocol, A can find this path and establish a flow to the base station. Let this path be A, B, C and the capacity of the three links be 1 for $(\mathrm{A}, \mathrm{B}), 2$ for $(\mathrm{B}, \mathrm{C})$ and 1.5 for (C, BS). Capacity can be any metric that is meaningful for the network, as long as the capacity of the entire path is defined as the minimum of the capacities of the links. In our example the capacity of the path is 1 . This means that $\mathrm{A}$ can receive data on this path at a rate decided by the capacity of the $(\mathrm{A}, \mathrm{B})$ link. This leaves links (B, C) and (C, BS) with a residual capacity. For simplicity, we assume for this example, that the residual capacity is the initial capacity, decreased by the path capacity, although this is not an assumption we use 
in the rest of the paper. In general, if every time a flow is added, the end host receives data at the maximum rate allowed by the path, a link will be saturated and that link will decide the capacity of the flow. As a result, after each time a flow is added or removed, we can deduce the state of the residual network from the physical state of the links and the sequence of flow additions and deletions.

Consequently, a routing protocol that maintains the optimal path for each host in the residual network can discover multihop paths by only keeping a parent pointer for each host. In our example, the parent of A is B, the parent of $B$ is $C$ and the parent of $C$ is the base station. After $A$ adds a flow, the parent information might have to change to reflect the decrease in the capacity of the three links. At all times, the routing information constitutes a spanning tree rooted at the base station. We can prove that the maximum spanning tree of a residual network provides the optimal routing information. This is a direct implication of the following well-known property of maximum spanning trees

Cycle property. For any cycle $\mathrm{C}$ in $\mathrm{G}$, the lightest edge in $\mathrm{C}$ does not appear in the maximum spanning tree.

In other words, we can construct a maximum spanning tree by deleting the worst link of every simple cycle in the network. As a result, the path from each host to the root in the spanning tree has the maximum minimum link possible, which maximizes the capacity of the entire path.

Given a maximum spanning tree, a host can schedule the next flow by sending a forward request to its parent, which in turn will forward the request to its parent, until the base station is reached. The problem is after adding (or deleting) a flow, the entire network may need to be contacted to derive the new maximum spanning tree, which is asymptotically not better than flooding each time a flow needs to be scheduled.

To solve the scalability issues, each time there is a change in the network, we can lazily converge to the maximum spanning tree, instead of trying to keep up with the changes. Our distributed spanning tree (DST) protocol does not change the routing information to correspond to the maximum spanning tree each time a flow is added or removed. However, each time a host requests a path, its parent pointer is set to the neighbor that has the optimal path to the base station, according to the existing information. The changes are confined in the neighborhood of a host and while queries about the state of the paths of the neighbors have to contact hosts outside the neighborhood, they can be completed much faster, as we will show. We note that if the network becomes static, the routing information will eventually converge to the optimal, even with this localized updating policy.

The advantage of this approach is that efficiently maintaining a spanning tree is possible even for large networks, as it requires only $\mathrm{O}(\log \mathrm{n})$ time and messages for each operation, the complexity of maintaining a dynamic tree. In the worst case, it can be arbitrarily far from the optimum, but our experiments indicate that, on average, this is not the case. For large and active networks, where nodes request flows frequently and links are close to capacity, DST performs extremely well.

\section{B. Maintaining a Dynamic Tree}

The entire routing protocol can be split into two layers communicating through a well-defined interface. The top layer is responsible for the maintenance of the distributed spanning tree. This layer issues a string of operations on the spanning tree. These operations are from the following set:

- $\operatorname{Link}(\mathrm{v}, \mathrm{u}, \mathrm{w})$ Merge the tree rooted at node $\mathrm{v}$ with the tree of node $u$ by making $u$ the parent of $v$. The weight of the new link is w.

- Cut(v) Split a tree into two by removing the link of node $\mathrm{v}$ to its parent.

- Mincost(v) Return the minimum weight cost edge on the path from node $\mathrm{v}$ to the root of the tree it belongs to. This operation is called for every neighbor of a node when a new parent has to be established.

- Root(v) Find the root of the tree $\mathrm{v}$ belongs to. This operation is useful in avoiding cycles when the Mincost operation is called by $u$ for a child $v$ in the spanning tree.

- Update $(\mathrm{v}, \mathrm{w})$ Add w to all edges on the path from $\mathrm{v}$ to the root of its tree. It is called to increase or reduce the capacity of a link after adding or removing a flow.

- Cost(v) Return the cost of the edge from v to its parent.

The second layer is responsible for efficiently completing these requests. We have chosen to implement this layer with a link-cut tree. Link-cut trees are structures that can complete the above described set of operations in $\mathrm{O}(\log \mathrm{n})$ time, where $\mathrm{n}$ is the number of hosts. This scalability property is important as it translates in $\mathrm{O}(\log \mathrm{n})$ messages when implemented distributedly. Furthermore, when a new host enters the network and has to query its neighbors on the capacity of their paths, the parallel time complexity is $\mathrm{O}(\log \mathrm{n})$. For implementation purposes we have chosen to use topology trees for this layer. A topology tree is a relatively simple link-cut tree and it has a natural distributed implementation. We note that in principle any dynamic tree can be used for this layer, as long as it does not modify the structure of the spanning tree. The root of the tree is fixed to be the base station and the links are oriented towards it. Balancing operations may have to change the root and the orientation of the links and cannot be used.

\section{Refresh Rate}

In dynamic networks, routes become stale quickly. A parent pointer indicating the best available path should be reevaluated at constant intervals to adapt to topology changes. The exact refresh rate depends on how dynamic the hybrid network is and how much traffic per operation we want to allow. The strategy we use for reassessing the parent pointer has a node cutting its parent every $\mathrm{k}$ seconds and probing all its neighbors. The traffic generated 
is $\mathrm{O}(\mathrm{d} \log \mathrm{n})$, where $d$ is the number of neighbors. The parent pointer is as close to the optimal as possible with the available information.

To keep the number of messages per time unit at a scalable level, we have to modulate $\mathrm{k}$ with the size of the network. As the network becomes more dense, the refresh rate should drop. If $\mathrm{k}=\Theta(\mathrm{d})$, the traffic generated every $k$ seconds is $\mathrm{O}(\mathrm{k} \log \mathrm{n})$. The exact constants depend on the specifics of the network, but the conclusion is that for dense networks, refreshing should be done more sparingly. This appears to be contrary to the scalability of DST, as the spanning tree maintained should be farther from the optimal. However, dense hybrid networks are more robust, and even if a link disappears, there is a high probability that an alternate equivalent link will be present. A parent pointer can be used with relative confidence for the short time until the next reassessment. In Section III, we use an alternate approach, scaling $\mathrm{k}$ with $\log \mathrm{n}$, which generates O(d) messages per time unit for each host. Even at this rate, the scalability of the network is not affected, as probing one's neighbors with a heartbeat broadcast, an operation performed by almost all wireless interfaces, generates d replies. Experiments confirm that dropping the refresh rate according to $\log \mathrm{n}$ does not affect the performance of DST.

\section{Simulation Results}

In our experiments we model the ad-hoc network using the unit disk graph model, where the transmission range of a host is 115 units. We use the ARF [3] mechanism to establish the bandwidth of the communication channel between two hosts. More precisely, the bandwidth is $11 \mathrm{Mbps}$ for distances under 50 units, $5.5 \mathrm{Mbps}$ at 70 units, $2 \mathrm{Mbps}$ at 90 units and $1 \mathrm{Mbps}$ at 115 units. The initial positions of the hosts are chosen randomly in a square of size $886 \times 886$ units. We use the random waypoint model [4] to simulate the movements of the hosts.

We use the dependency between the HDR downlink rate and the distance from the base station depicted in [1], to model the cellular downlink rate of hosts. The base station is positioned in the center of the square and each mobile host inside the square is covered by the cellular transmission range of the base station.

We used the Bellman-Ford algorithm to compute the maximum capacity paths. In the case of multiple concurrent flows the optimal for the $\mathrm{n}$-th flow is computed after removing the bandwidth consumed by the first $\mathrm{n}-1$ flows.

Figure 1 shows the performance of DST and UCAN relative to the optimal total throughput, achieved when all the client hosts run the distributed Bellman-Ford algorithm to find the best downlink path. The results are shown for configurations of 100 hosts, when the number of concurrent flows, initiated by different clients, grows from 1 to 50. UCAN achieves its maximum performance, $70 \%$, for 2 concurrent flows, but then it constantly decreases, reaching as low as $40 \%$ of the optimum. DST consistently outperforms UCAN, with a maximum of $90 \%$ of the optimum for a single flow, and a minimum of $70 \%$ for 15 concur-

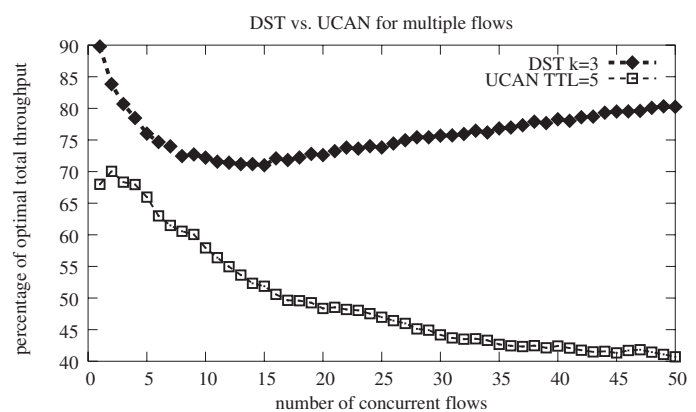

Fig. 1. The throughput of DST and UCAN as percentage of the optimal value for networks of 100 nodes, when the number of concurrent flows grows from 1 to 50 .

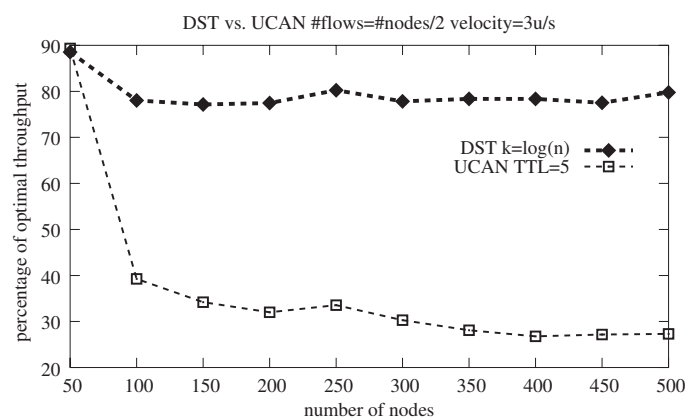

Fig. 2. The throughput of DST and UCAN as percentage of the optimal value for networks of up to 500 hosts, when the number of concurrent flows is half the number of hosts. The refresh rate of DST is set to $\log \mathrm{n}$, where $\mathrm{n}$ is the number of hosts.

rent flows, but recovering after it and reaching $80 \%$ of the optimum for 50 concurrent flows.

The second experiment evaluates DST and UCAN when the total number of hosts increases from 50 to 500 , and for each configuration, half of the hosts have a flow. Again, for DST, the value of $k$ is set to be $\log n$, where $n$ is the total number of hosts. Figure 2 shows the percentage of the optimal total throughput achieved by DST and UCAN. For a small total number of hosts, 50, DST and UCAN have similar performance, close to $90 \%$. However, UCAN's performance decreases constantly with the number of hosts, reaching under $30 \%$ for 500 hosts, whereas DST stabilizes at around $80 \%$ of the optimum.

\section{REFERENCES}

[1] Haiyun Luo, Ramachandran Ramjee, Prasun Sinha, Li (Erran) Li, and Songwu Lu. Ucan: a unified cellular and ad-hoc network architecture. In Proceedings of the 9th annual international conference on Mobile computing and networking, pages 353-367. ACM Press, 2003.

[2] G. N. Frederickson. A data structure for dynamically maintaining rooted trees. In SODA, 1993.

[3] A. Kamerman and L. Monteban. Wavelan-ii: A high performance wireless lan for the unlicensed band. Bell Labs Technical Journal, 1997.

[4] D.B. Johnson and D.A. Maltz. Dynamic source routing in ad hoc wireless networks. In Mobile Computing, volume 353, pages 153-181. Kluwer Academic Publishers, 1996. 